\title{
Local unitary equivalence of multipartite pure states
}

\author{
B. Kraus \\ Institute for Theoretical Physics, University of Innsbruck, Austria
}

\begin{abstract}
Necessary and sufficient conditions for the equivalence of arbitrary $n$-qubit pure quantum states under Local Unitary (LU) operations are derived. First, an easily computable standard form for multipartite states is introduced. Two generic states are shown to be LU-equivalent iff their standard forms coincide. The LU-equivalence problem for non-generic states is solved by presenting a systematic method to determine the LU operators (if they exist) which interconvert the two states.
\end{abstract}

Multipartite states occur in many applications of quantum information, like one-way quantum computing, quantum error correction, and quantum secret sharing $[1,2]$. Furthermore, the theory of multipartite states plays also an important role in other fields of physics which deal with many-body systems [3]. The existence of those practical and abstract applications is due to the subtle properties of multipartite entangled states. Thus, one of the main goals in quantum information theory is to gain a better understanding of the non-local properties of quantum states. Whereas the bipartite case is well understood, the multipartite case is much more complex. Even though a big theoretical effort has been undertaken where several entanglement measures for multipartite states have been introduced [4], different classes of entangled states have been identified [5], and a normal form of multipartite states has been presented [6], we are far from completely understanding the non-local properties of multipartite states [7].

One approach to gain insight into the entanglement properties of quantum states is to consider their interconvertability. That is, given two states $|\Psi\rangle,|\Phi\rangle$ the question is whether or not $|\Psi\rangle$ can be transformed into $|\Phi\rangle$ by local operations [7]. One particularly interesting case, which is also investigated in this paper, is the LU-equivalence of multipartite states. We say that a $n^{-}$ partite state, $|\Psi\rangle$ is LU-equivalent to $|\Phi\rangle\left(|\Psi\rangle \simeq_{L U}|\Phi\rangle\right)$ if there exist local unitary operators, $U_{1}, \ldots, U_{n}$, such that $|\Psi\rangle=U_{1} \otimes \cdots \otimes U_{n}|\Phi\rangle$. Note that two states which are LU-equivalent are equally useful for any kind of application and they posses precisely the same amount of entanglement. This is why understanding the interconvertability of quantum states by $\mathrm{LU}$ operations is part of the solution to the more general problem of characterizing the different types of entangled quantum states.

In order to solve this long-standing problem the socalled local polynomial invariants have been introduced [8]. However, even though it is known that it is sufficient to consider only a finite set of them, this complete finite set is known only for very few simple cases.

Here, we derive necessary and sufficient conditions for the existence of LU operations which transform two states into each other. For generic states, states where non of the single qubit reduced states is completely mixed, the conditions can be easily computed. For arbitrary $n$-qubit states a systematic method to determine the unitaries (in case they exist) which interconvert the states is presented.

The sequel of the paper is organized as follows. First, we introduce a standard form of multipartite states, which we use in order to derive easily computable necessary and sufficient conditions for the LU-equivalence of generic multipartite states. Like in the bipartite case, it is shown that two generic states are LU-equivalent iff their standard forms coincide. For non-generic states it is shown that whenever one of the single qubit reduced states is not completely mixed, the problem of LU-equivalence of $n$-qubit states can be reduced to the problem of LU-equivalence of $(n-1)$-qubit states. Then, a systematic method to determine the local unitaries (if they exist) which interconvert two arbitrary states is presented. It is shown that the states are LU-equivalent iff there exists a solution to a finite set of equations. The number of variables involved in those equations depends on the entanglement properties of the states. The case with the largest number of variables occurs for the sometimes called maximally entangled states of $n$ qubits, where any bipartition of $\lceil n / 2\rceil$ qubits is maximally entangled with the rest. It is known however, that only for certain values of $n$ such states exist [9]. The power of this method is illustrated by considering several examples.

Throughout this paper the following notation is used. By $X, Y, Z$ we denote the Pauli operators. The subscript of an operator will always denote the system it is acting on, or the system it is describing. The reduced states of system $i_{1}, \ldots i_{k}$ of $|\Psi\rangle(|\Phi\rangle)$ will always be denoted by $\rho_{i_{1} \ldots i_{k}}\left(\sigma_{i_{1} \ldots i_{k}}\right)$ resp., i.e. $\rho_{i_{1} \ldots i_{k}}=\operatorname{tr}_{\neg i_{1} \ldots \neg i_{k}}(|\Psi\rangle\langle\Psi|)$. We denote by $\mathbf{i}$ the classical bit-string $\left(i_{1}, \ldots, i_{n}\right)$ with $i_{k} \in\{0,1\} \forall k \in\{1, \ldots, n\}$ and $|\mathbf{i}\rangle \equiv\left|i_{1}, \ldots, i_{n}\right\rangle$ denotes the computational basis. Normalization factors as well as the tensor product symbol will be omitted whenever it does not cause any confusion.

Let us start by introducing a unique standard form of multipartite states (see also [10]). Let $|\Psi\rangle$ be a $n-$ qubit state. As a first step we apply local unitaries, $U_{i}^{1}$ such that all the single qubit reduced states of the state $\left|\Psi_{t}\right\rangle=$ $U_{1}^{1} \otimes \ldots U_{n}^{1}|\Psi\rangle$ are diagonal in the computational basis, i.e. $\operatorname{tr}_{\neg i}\left(\left|\Psi_{t}\right\rangle\left\langle\Psi_{t}\right|\right)=D_{i}=\operatorname{diag}\left(\lambda_{i}^{1}, \lambda_{i}^{2}\right)$. We call any such decomposition trace decomposition of the state $|\Psi\rangle$. A sorted trace decomposition is then defined as a trace decomposition with $\lambda_{i}^{1} \geq \lambda_{i}^{2}$. Note that transforming a state into its sorted trace decomposition, which we will denote by $\left|\Psi_{s t}\right\rangle$ in the following, can be easily done by computing the spectral decomposition of all the single 
qubit reduced states. The sorted trace decomposition of a generic state, $|\Psi\rangle$ with $\rho_{i} \neq \mathbb{1} \forall i$ is unique up to local phase gates. That is $U_{1} \ldots U_{n}\left|\Psi_{s t}\right\rangle$ is a sorted trace decomposition of $|\Psi\rangle$ iff (up to a global phase, $\alpha_{0}$ ) $U_{i}=$ $U_{i}\left(\alpha_{i}\right) \equiv \operatorname{diag}\left(1, e^{i \alpha_{i}}\right)$. In order to make the sorted trace decomposition of generic states unique we impose the following condition on the phases $\alpha_{i}, i \in\{0, \ldots, n\}$. We write $\left|\Psi_{s t}\right\rangle=\sum_{i_{1}, \ldots i_{n}=0}^{1} \lambda_{i_{1}, \ldots, i_{n}}\left|i_{1}, \ldots, i_{n}\right\rangle$, and define the set $S=\left\{\mathbf{i}: \lambda_{\mathbf{i}} \neq 0\right\}$ and $\bar{S}$ denotes the set of the linearly independent vectors in $S$. The global phase, $\alpha_{0}$ is chosen to make $\lambda_{\mathbf{i}_{\mathbf{o}}}$ real and positive where $\mathbf{i}_{\mathbf{0}}=\mathbf{0}$ in case $\lambda_{\mathbf{0}} \neq 0$ else $\mathbf{i}_{\mathbf{0}}$ denotes the first (in lexicographic order) linearly dependent vector in $S$. After that, the $n$ phases are chosen to make the coefficients $e^{i \alpha_{0}} \lambda_{\mathbf{i}}$ for $\mathbf{i} \in \bar{S}$ real and positive [13]. Since all the phase gates, which do not leave the state invariant are fixed in this way we have that $U_{1} \ldots U_{n}\left|\Psi_{s}\right\rangle$, where $\left|\Psi_{s}\right\rangle$ denotes here and in the following the standard form of $|\Psi\rangle$, has standard form iff $U_{1} \ldots U_{n}\left|\Psi_{s}\right\rangle=\left|\Psi_{s}\right\rangle$. That is the standard form is unique. If $\rho_{i}=\frac{1}{2} \mathbb{1}$, for some system $i$, the standard form can be similarly defined [10], however it will not be unique then. Due to the definition any state is LU-equivalent to its standard form [14].

We employ now the standard form to derive a criterion for the LU-equivalence of generic multipartite states. First of all note that $|\Psi\rangle \simeq_{L U}|\Phi\rangle$ iff $\left|\Psi_{s}\right\rangle \simeq_{L U}\left|\Phi_{s}\right\rangle$. Using then that the standard form is unique we obtain the following theorem.

Theorem 1. Let $|\Psi\rangle$ be an $n$ qubit state with $\rho_{i} \neq \mathbb{1}$ $\forall i$. Then $|\Psi\rangle \simeq_{L U}|\Phi\rangle$ iff the standard form of $|\Psi\rangle$ is equivalent to the standard form of $|\Phi\rangle$, i.e. $\left|\Psi_{s}\right\rangle=\left|\Phi_{s}\right\rangle$.

Thus, similarly to the bipartite case, two generic states are LU-equivalent iff their standard forms coincide, which can be easily checked. Furthermore, if the states are LU-equivalent then $|\Psi\rangle=U_{1}, \ldots, U_{n}|\Phi\rangle$ with $U_{i}=\left(U_{s}^{i}\right)^{\dagger} V_{s}^{i}$, where $U_{s}^{i}, V_{s}^{i}$ denote the local unitaries such that $\left|\Psi_{s}\right\rangle \equiv U_{s}^{1} \ldots U_{s}^{n}|\Psi\rangle$ and $\left|\Phi_{s}\right\rangle \equiv V_{s}^{1} \ldots V_{s}^{n}|\Phi\rangle$.

In order to study now the non-generic cases, we will rewrite the necessary and sufficient condition derived above. For a generic state, $|\Psi\rangle$ it is easy to verify that $\left|\Psi_{s}\right\rangle=\left|\Phi_{s}\right\rangle$ iff there exists a bitstring $\mathbf{k}=k_{1}, \ldots k_{n}$, local phase gates $U_{i}\left(\alpha_{i}\right)$, and a global phase $\alpha_{0}$ s.t.

$$
e^{i \alpha_{0}} \bigotimes_{i} U_{i}\left(\alpha_{i}\right) X_{i}^{k_{i}} \bar{W}_{i}|\Psi\rangle=\bigotimes_{i} \bar{V}_{i}|\Phi\rangle,
$$

where $\bar{W}_{i}\left(\bar{V}_{i}\right)$ are local unitaries which transform $\rho_{i}$ $\left(\sigma_{i}\right)$ into a diagonal matrix. That is $\bigotimes_{i} \bar{W}_{i}|\Psi\rangle$ and $\bigotimes_{i} \bar{V}_{i}|\Phi\rangle$ are trace decompositions of $|\Psi\rangle$ and $|\Phi\rangle$ resp.. For generic states $k_{i}$ is chosen such that the order of the eigenvalues of the single qubit reduced states of $\bigotimes_{i} X_{i}^{k_{i}} \bar{W}_{i}|\Psi\rangle$ and $\bigotimes_{i} \bar{V}_{i}|\Phi\rangle$ coincides. In order to check then whether or not there exist phases $\alpha_{i}$ such that Eq. (1) is satisfied, we make use of the following lemma. There, we will consider four $n-$ qubit states. The systems, each composed out of $n$ qubits will be denoted by $A, B, C, D$ respectively. The $i$-th qubit of system
$A$ will be denoted by $A_{i}$, etc. Furthermore, we will use the notation $\left|\chi_{i}\right\rangle=(|0110\rangle-|1001\rangle)_{A_{i}, B_{i}, C_{i}, D_{i}}$ and $P_{A C}^{i}=\sum_{\mathbf{k}}|\mathbf{k}\rangle\left\langle\left.\mathbf{k} \mathbf{k}\right|_{A_{1}, C_{1}, \ldots A_{i-1}, C_{i-1}, A_{i+1}, C_{i+1} \ldots, A_{n}, C_{n}}\right.$ and similarly we define $P_{B D}^{i}$ for systems $B, D$. For a state $|\Psi\rangle$ we define $K_{\Psi} \equiv\{\mathbf{k}$ such that $\langle\mathbf{k} \mid \Psi\rangle=0\}$ and $\left|\Psi_{\bar{\alpha}_{i}}\right\rangle=|\Psi\rangle+e^{-i \bar{\alpha}_{0}} \sum_{\mathbf{k} \in K_{\Psi}} e^{-i \sum_{i=1}^{n} \bar{\alpha}_{i} k_{i}}|\mathbf{k}\rangle$ for some phases $\bar{\alpha}_{i}$ and $\left|\Psi_{\mathbf{0}}\right\rangle=|\Psi\rangle+\sum_{\mathbf{k} \in K_{\Psi}}|\mathbf{k}\rangle$.

Lemma 2. Let $|\Psi\rangle,|\Phi\rangle$ be $n$ qubit states. Then, there exist local phase gates, $U_{i}\left(\alpha_{i}\right)$ and a phase $\alpha_{0}$ such that $|\Psi\rangle=e^{i \alpha_{0}} \bigotimes_{i=1}^{n} U_{i}\left(\alpha_{i}\right)|\Phi\rangle$ iff there exist phases $\left\{\bar{\alpha}_{i}\right\}_{i=0}^{n}$ such that

$$
\begin{aligned}
& \text { (i) } \left.\left|\left\langle\mathbf{i} \mid \Psi_{\mathbf{0}}\right\rangle\right|=\left|\langle\mathbf{i}| \Phi_{\bar{\alpha}_{i}}\right)\right\rangle \mid \forall \mathbf{i} \text { and } \\
& \text { (ii) }\left\langle\left.\chi\right|_{i} P_{A C}^{i} P_{B D}^{i} \mid \Psi_{\mathbf{0}}\right\rangle_{A}\left|\Psi_{\mathbf{0}}\right\rangle_{B}\left|\Phi_{\bar{\alpha}_{i}}\right\rangle_{C}\left|\Phi_{\bar{\alpha}_{i}}\right\rangle_{D}=0 \forall i \in \\
& \{1, \ldots, n\} .
\end{aligned}
$$

The prove of this lemma will be presented in the appendix.

Let us now consider the non-generic case. Obviously, two arbitrary states, $|\Psi\rangle,|\Phi\rangle$, are LU-equivalent iff there exist local unitaries $\bar{V}_{k}, \bar{W}_{k}$ a bit string $\mathbf{k}$ and phases $\alpha_{i}$ such that Eq. (1) is fulfilled. We will show now how $\bar{V}_{k}, \bar{W}_{k}$ can be determined by imposing necessary conditions of LU-equivalence.

First of all, we note that for any state $|\Psi\rangle$ with $\rho_{i} \neq \mathbb{1}$ for some system $i, k_{i}$ as well as $\bar{V}_{i}$ and $\bar{W}_{i}$ can be easily determined as follows. If $|\Psi\rangle \simeq_{L U}|\Phi\rangle$ then all the reduced states must be LU-equivalent, in particular $D_{i}=\operatorname{diag}\left(\lambda_{1}^{i}, \lambda_{2}^{i}\right)=\bar{W}_{i} \rho_{i} \bar{W}_{i}^{\dagger}=\bar{V}_{i} \sigma_{i} \bar{V}_{i}^{\dagger}$, for some unitaries $\bar{W}_{i}, \bar{V}_{i}$. Analogously to the generic case, this equation determines $\bar{W}_{i}$ and $\bar{V}_{i}$ (and $k_{i}=0$ ) uniquely up to a phase gate. Thus, for this case we have that $|\Psi\rangle \simeq_{L U}|\Phi\rangle$ iff there exist two phases, $\alpha_{i}$ and $\alpha_{0}$ and local unitaries $U_{j}$ such that

$$
{ }_{i}\left\langle l \mid \bar{W}_{i} \Psi_{s}\right\rangle=e^{i\left(\Phi+\alpha_{i} l\right)} \bigotimes_{j \neq i} U_{j i}\left\langle l \mid \bar{V}_{i} \Phi_{s}\right\rangle,
$$

where $l \in\{0,1\}$ and $\bar{W}_{i}$, and $\bar{V}_{i}$ are chosen such that $D_{i}=\operatorname{diag}\left(\lambda_{1}^{i}, \lambda_{2}^{i}\right)=\bar{W}_{i} \rho_{i} \bar{W}_{i}^{\dagger}=\bar{V}_{i} \sigma_{i} \bar{V}_{i}^{\dagger}$. Hence, if there is one system where the reduced state is not proportional to the identity then we can reduce the problem of LUequivalence of $n$-qubit states to the LU-equivalence of $(n-1)$-qubit states. This statement can be easily generalized to the case where more than one single qubit reduced state is not completely mixed.

Let us now consider the more complicated case, where some $\rho_{i}=\mathbb{1}$. There, it is obviously no longer possible to determine $\bar{V}_{i}, \bar{W}_{i}$ by imposing the necessary condition of LU-equivalence, $\rho_{i}=U_{i} \sigma_{i} U_{i}^{\dagger}$. However, we will show next, which necessary condition can be used in order to determine them. Before we do so, we explain the problem which might occur if $\rho_{i}=\mathbb{1}$ by considering a simple example. Let $|\Psi\rangle$ and $|\Phi\rangle$ denote two states with $\rho_{12}=\sigma_{12}=\mathbb{1}-\lambda\left|\Psi^{-}\right\rangle\left\langle\Psi^{-}\right|$, for some $\lambda \neq 0$. Then we find that $\rho_{12}=U_{1} U_{2} \sigma_{12} U_{1}^{\dagger} U_{2}^{\dagger}$ iff $U_{1}=U_{2}$, which implies that $|\Psi\rangle \simeq_{L U}|\Phi\rangle$ iff there exist local unitaries 
$U_{1}, U_{3}, \ldots, U_{n}$ such that $|\Psi\rangle=U_{1} U_{1} \ldots U_{n}|\Phi\rangle$. Thus, the unitary $U_{2}$ depends on $U_{1}$. Or, stated differently, $\bar{W}_{2}$ (and $\alpha_{2}$ ) depends on $U_{1}$ in Eq. (1), where we set $V_{1}=V_{2}=\mathbb{1}$. In general we might neither be able to determine the phase $\alpha_{2}$, nor $\bar{W}_{2}$ as a function of $U_{1}$ alone. However, the next lemma shows that any $\bar{W}_{k}$ can be determined as a function of a few unitaries and $\bar{V}_{k}$ can always be determined directly form the state $|\Phi\rangle$. We will see that the number of unitaries which are required to define $\bar{W}_{k}$ depends on the entanglement properties of the state.

Lemma 3. If $|\Psi\rangle=U_{1} \ldots U_{n}|\Phi\rangle$ and if there exist systems $i_{1}, \ldots i_{l}$ such that $\rho_{i_{1}, \ldots i_{l}, k} \neq \rho_{i_{1}, \ldots i_{l}} \otimes \mathbb{1}$ then $\bar{V}_{k}$ in $E q$. (1) can be determined from the state $|\Phi\rangle$ and $\bar{W}_{k}$ can be determined as a function of $U_{i_{1}}, \ldots U_{i_{l}}$.

Proof. Without loss of generality we assume $i_{1}=$ $1, \ldots i_{l}=l$ and write $|\Psi\rangle=\sum|\mathbf{i}\rangle_{1 \ldots, l}\left|\Psi_{\mathbf{i}}\right\rangle_{l+1 \ldots, n}$ and $|\Phi\rangle=\sum|\mathbf{i}\rangle_{1 \ldots, l}\left|\Phi_{\mathbf{i}}\right\rangle_{l+1 \ldots, n}$, where $\mathbf{i}=\left(i_{1}, \ldots, i_{l}\right)$. Since $\sigma_{1, \ldots, l, k}=\sum|\mathbf{i}\rangle\langle\mathbf{j}| \operatorname{tr}_{\neg k}\left(\left|\Phi_{\mathbf{i}}\right\rangle\left\langle\Phi_{\mathbf{j}}\right|\right) \neq \sigma_{1, \ldots, l} \otimes \mathbb{1}$, there exist at least two tuples $\mathbf{i}$ and $\mathbf{j}=\left(j_{1}, \ldots j_{l}\right)$ such that the $2 \times 2$ matrix $X_{\mathbf{i}}^{\mathbf{j}} \equiv \operatorname{tr}_{\neg k}\left(\left|\Phi_{\mathbf{i}}\right\rangle\left\langle\Phi_{\mathbf{j}}\right|\right) \not \subset \mathbb{1}$. Thus, at least one of the two hermitian operators $Y_{\mathbf{i}}^{\mathbf{j}}=X_{\mathbf{i}}^{\mathbf{j}}+\left(X_{\mathbf{i}}^{\mathbf{j}}\right)^{\dagger}$ and $Z_{\mathbf{i}}^{\mathbf{j}}=$ $i X_{\mathbf{i}}^{\mathbf{j}}-i\left(X_{\mathbf{i}}^{\mathbf{j}}\right)^{\dagger}$ is not proportional to the identity. W. l. o. g. we assume that $\mathbb{1} \not \subset Y_{\mathbf{i}}^{\mathbf{j}}=\operatorname{tr}_{\neg k}[(|\mathbf{i}\rangle\langle\mathbf{j}|+h . c)|\Phi\rangle\langle\Phi|]$. Using that $|\Psi\rangle=U_{1} \ldots U_{n}|\Phi\rangle$ we have

$U_{k} Y_{\mathbf{i}}^{\mathbf{j}} U_{k}^{\dagger}=\operatorname{tr}_{\neg k}\left[(|\mathbf{i}\rangle\langle\mathbf{j}|+h . c) \cdot U_{1}^{\dagger} \ldots U_{l}^{\dagger}|\Psi\rangle\langle\Psi| U_{1} \ldots U_{l}\right] .(3)$

Since $Y_{\mathbf{i}}^{\mathbf{j}}$ is hermitian we can diagonalize it as well as the right hand side of Eq (3) and obtain $U_{k} \bar{V}_{k}^{\dagger} D \bar{V}_{k} U_{k}^{\dagger}=$ $\bar{W}_{k}^{\dagger}\left(U_{1}, \ldots U_{l}\right) D\left(U_{1}, \ldots U_{l}\right) \bar{W}_{k}\left(U_{1}, \ldots U_{l}\right)$, which is true iff $D=X^{i_{k}} D\left(U_{1}, \ldots U_{l}\right) X^{i_{k}}$, with $i_{k} \in\{0,1\}$ and $U_{k}=$ $e^{i \alpha_{0}} \bar{W}_{k}^{\dagger}\left(U_{1}, \ldots U_{l}\right) U\left(\alpha_{k}\right) X^{i_{k}} \bar{V}_{k}$, for some phases $\alpha_{0}, \alpha_{k}$. Note that Eq (3) must hold for any $\mathbf{i}, \mathbf{j}$. Note further that $\bar{V}_{k}$ is the unitary which diagonalizes $Y_{\mathbf{i}}^{\mathbf{j}}$ and can therefore be determined directly from the state $|\Phi\rangle$. Thus, we have $|\Psi\rangle=U_{1} \ldots U_{n}|\Phi\rangle$ iff there exists $i_{k} \in\{0,1\}$, $\alpha_{0}$ and $\alpha_{k}$ such that $e^{i \alpha_{0}} X^{i_{k}} U\left(\alpha_{k}\right) \bar{W}_{k}\left(U_{1}, \ldots, U_{l}\right)|\Psi\rangle=$ $U_{1} \ldots \bar{V}_{k} \ldots U_{n}|\Phi\rangle$.

Note that the proof of Lemma 3 is constructive. The idea was to impose the necessary condition for LUequivalence given in Eq. (3) for any $l$-tuples $\mathbf{i}, \mathbf{j}$. Since the $2 \times 2$ matrices occurring in this equation are hermitian, one can, similarly to the previous cases, determine the unitaries $\bar{V}_{k}, \bar{W}_{k}$ by diagonalizing these matrices. In contrast to before we will find here, that $\bar{W}_{k}$ might depend on $U_{1}, \ldots, U_{l}$.

We use now Lemma 3 to present a constructive method to compute all local unitaries as functions of a few variables. If some unitary, $U_{i}$ cannot be determined in this way, we write $U_{i}=e^{-i \gamma_{i} Z_{i}} e^{-i \beta_{i} X_{i}} e^{-i \alpha_{i} Z_{i}}$ (up to a phase). Then $|\Psi\rangle=\otimes_{j} U_{j}|\Phi\rangle$ iff $e^{i \alpha_{i} Z_{i}} \bar{W}_{i}|\Psi\rangle=\otimes_{j \neq i} U_{j}|\Phi\rangle$, where $\bar{W}_{i}=e^{i \beta_{i} X_{i}} e^{i \gamma_{i} Z_{i}}$. That is, in this case we set $\bar{V}_{i}=\mathbb{1}, k_{i}=0$, and $\bar{W}_{i}=e^{i \beta_{i} X_{i}} e^{i \gamma_{i} Z_{i}}$ in Eq (1). We will say then that we consider $U_{i}$ as a variable.
The constructive method to compute now $\bar{V}_{k}$ and $\bar{W}_{k}$ in Eq (1) is as follows: (1) If there exists a system $i$ such that $\rho_{i} \not \propto \mathbb{1}$ compute $\bar{V}_{i}, \bar{W}_{i}$ using that $\bar{W}_{i} \rho_{i} \bar{W}_{i}^{\dagger}=\bar{V}_{i} \sigma_{i} \bar{V}_{i}^{\dagger}$ $\left(k_{i}=0\right)$. Furthermore, compute $\bar{V}_{k}$ and $\bar{W}_{k}\left(U_{i}\right)$ for any system $k$ with $\rho_{i k} \neq \rho_{i} \otimes \mathbb{1}$ using Lemma 3. (2) For all systems $i$ for which $\rho_{i} \neq \mathbb{1}$ apply the unitaries $\bar{W}_{i}\left(\bar{V}_{i}\right)$ to $|\Psi\rangle(|\Phi\rangle)$ resp. and measure system $i$ in the computational basis thereby reducing the number of systems (see Eq (2)). After this step we have $\rho_{i} \propto \mathbb{1} \forall i$. Then we continue as follows: (3) Consider the two qubit reduced states: (3a) There exist systems $i, j$ such that $\rho_{i j} \neq \mathbb{1}$. W. l. o. g. we choose $i=1$, consider $U_{1}$ as variable, and set $\bar{V}_{1}=\mathbb{1}, k_{1}=0$ and $\bar{W}_{1}=e^{i \beta_{1} X_{1}} e^{i \gamma_{1} Z_{1}}$. Then, compute $\bar{V}_{j}$ and $\bar{W}_{j}\left(U_{1}\right)$ using Lemma 3 for any system $j$ with $\rho_{1 j} \not \subset \mathbb{1}$. Let us denote by $J_{2}$ the set of systems for which $\rho_{1 j} \not \subset \mathbb{1}$. (3b) If there exists no system $i, j$ such that $\rho_{i j} \not \notin \mathbb{1}$ consider $U_{1}$ and $U_{2}$ as variables and set $\bar{V}_{i}=\mathbb{1}, k_{i}=0$ and $\bar{W}_{i}=e^{i \beta_{i} X_{i}} e^{i \gamma_{i} Z_{i}}$, for $i=1,2$. Furthermore, set $J_{2}=\{2\}$. (4) Consider the three-qubit reduced states: (4a) If there exists a system $k$ such that $\rho_{1 j k} \neq \rho_{1 j} \otimes \mathbb{1}$ for some $j \in J_{2}$ compute $\bar{V}_{k}$ and $\bar{W}_{k}\left(U_{1}, U_{j}\right)$ using Lemma 3 . Determine for any system $k$ with $\rho_{1 j k} \neq \rho_{1 j} \otimes \mathbb{1} \bar{V}_{k}$ and $\bar{W}_{k}\left(U_{1}, U_{j}\right)$ (if they are not already determined). (4b) If there exists no system $k$ such that $\rho_{1 j k} \neq \rho_{1 j} \otimes \mathbb{1}$ include $U_{3}$ as variable. (5) Continue in this way until all unitaries are either determined as functions of a few unitaries, or are free parameters. If at some point it is not possible to choose $\bar{V}_{k}$ or $\bar{W}_{k}$ unitary, e.g. if the eigenvalues of the operators occurring in Eq (3) do not coincide, the states are not LU-equivalent.

Once all unitaries, $\bar{V}_{i}$ are determined and all unitaries $\bar{W}_{i}$ are determined as functions of a few variables, we have that $|\Psi\rangle \simeq_{L U}|\Phi\rangle$ iff there exists a bitstring $\mathbf{k}$ and phases $\left\{\alpha_{i}\right\}_{i=0}^{n}$, such that Eq (1) is fulfilled. In order to check the existence of the local phase gates in Eq. (1) (for some bitstring $\mathbf{k}$ ), we use Lemma 2. It is important to note here that the state on the right hand side of Eq. (1) is completely determined, thus, the set $K_{\Psi}$ in Lemma 2 can be determined and therefore this lemma can be applied. The states are LU-equivalent iff the conditions in Lemma 2 are fulfilled for some bitstring $\mathbf{k}$. Note that the unitaries, $U_{i}$ which transform $|\Phi\rangle$ into $|\Psi\rangle$ are then given by $U_{i}=\bar{W}_{i}^{\dagger} U\left(\alpha_{i}\right) X^{k_{i}} \bar{V}_{i}$ (up to a global phase) [15]. These unitaries are uniquely determined up to the symmetry of the state.

Note that a pure state has the property that $\rho_{i_{1}, \ldots, i_{l}, k}=\rho_{i_{1}, \ldots, i_{l}} \otimes \mathbb{1}_{k}$ iff for any outcome of any Von Neumann measurement on systems $i_{1}, \ldots, i_{l}$, system $k$ is maximally entangled with the remaining systems. Only in this case we have to add another unitary as a variable. It is clear that two states, $|\Psi\rangle,|\Phi\rangle$ with $\rho_{i_{1}, \ldots, i_{l}, k}=\rho_{i_{1}, \ldots, i_{l}} \otimes \mathbb{1}$ and $\sigma_{i_{1}, \ldots, i_{l}, k} \neq \sigma_{i_{1}, \ldots, i_{l}} \otimes \mathbb{1}$ can neither be LU-equivalent nor posses the same entanglement. Thus, the method presented above suggests that in order to characterize the non-local properties of multipartite states, one should first identify the class (as described above) to which the state belongs to and then determine within this class the entanglement of the state. It might 
well be, that the different classes lead to different applications. For instance, the states used for error correction, one way quantum computing and quantum secret sharing have the property that all single qubit reduced states are completely mixed.

Before we consider now some examples, let us mention that the worst case, i.e. the case which involves the largest number of variables, is the one where the reduced state of any bipartite splitting of $\lceil n / 2\rceil$ systems versus the rest are maximally mixed. In this case we have $\lceil n / 2\rceil$ unitaries as variables. Note however, that there are very few instances, where those states do exist [9].

In order to illustrate the power of this method we consider first the simplest examples of two and three qubit states. The standard form of a two qubit state is $|\Psi\rangle=$ $\lambda_{1}|00\rangle+\lambda_{2}|11\rangle$. Thus, the method above tells us that if $\lambda_{1} \neq \lambda_{2}$, i.e. $\rho_{i} \neq \mathbb{1}$, then, $|\Psi\rangle \simeq_{L U}|\Phi\rangle$ iff the Schmidt coefficients $\lambda_{i}$ are the same. For $\lambda_{1}=\lambda_{2}$ it is straightforward to show that the unitaries, $U_{i}$, which are obtained using the method above for the states $\left|\Phi^{+}\right\rangle \equiv|00\rangle+|11\rangle$ and some LU-equivalent state $V_{1} V_{2}\left|\Phi^{+}\right\rangle$are $U_{1}=V_{1} W$ and $U_{2}=V_{2} W^{*}$ for any unitary $W$. The reason why the unitaries $U_{i}$ are not completely determined by $V_{i}$ is due to the symmetry of the state, $\left|\Phi^{+}\right\rangle=W \otimes W^{*}\left|\Phi^{+}\right\rangle \forall W$ unitary.

For three qubits the method is almost equally simple. First, we transform both states into their trace decomposition. If non of the reduced states is completely mixed, we simply compare their standard forms (Theorem 1). If there exists some $i$ such that $\rho_{i} \neq \mathbb{1}$, we know that $U_{i}=U\left(\alpha_{i}\right)$. We measure system $i$ in the computational basis and are left with two two-qubit states (see Eq. (2)). In case those states are LU-equivalent we apply the corresponding unitaries and use Lemma 2 to find out whether the three qubit states are LU-equivalent or not. For the remaining case, where $\rho_{i}=\mathbb{1} \forall i$ it can be easily shown that $|\Psi\rangle$ is LU-equivalent to the GHZ-state, $\left|\Psi_{0}\right\rangle=|000\rangle+|111\rangle[12]$. Even without using this fact it can be easily shown that also in this case the method presented above leads directly to the right unitaries (up to the symmetry of the states) for two states which are LU-equivalent (for details see [12]).

With the same method the LU-equivalence classes of up to 5 -qubit states are investigated in [12]. We will show there, for instance, that for 4-qubit states with $\rho_{i j}=\mathbb{1}$ for some $i, j$ (which is the hardest class of states using the method presented above), the LU-equivalence class is determined by only three parameters. Thus, also the entanglement of those states is completely determined by the fact that system $i j$ is maximally entangled to the other two qubits and those three parameters, to which also an operational meaning will be given [12]. This example shows already that the method presented here does not only give necessary and sufficient conditions for the LU-equivalence of arbitrary multipartite states, but also leads to a new insight into their entanglement properties.

Finally, let us note that the results presented above serve also as a criterion of LU-equivalence for certain mixed and also $d$-level states. For instance, if there exists at least one non-degenerate eigenvalue of $\rho(\sigma)$ with corresponding eigenvectors $|\Psi\rangle(|\Phi\rangle)$ resp., then $\rho \simeq_{L U} \sigma$ implies that $|\Psi\rangle \simeq_{L U}|\Phi\rangle$. Using the method presented here, all the unitaries, which transform $|\Psi\rangle$ into $|\Phi\rangle$ can be determined and therefore it is straightforward to check if one of them also converts $\rho$ into $\sigma$.

In summary, a systematic way to show the LUequivalence of arbitrary multipartite pure states is presented. The results derived here also lead to a new insight into the entanglement properties of the multipartite states. Studying the different classes specified here, allows one to identify new parameters characterizing entanglement [12]. In particular, for generic states all the parameters occurring in the standard form determine, like in the bipartite case, the entanglement contained in the state.

The author would like to thank Hans Briegel for continuous support and interest in this work and acknowledges support of the FWF (Elise Richter Program).

\section{APPENDIX: INTERCONVERTABILITY BY LOCAL PHASE GATES}

In order to prove Lemma 2 we will make use of the following lemma, where we use the same notation as before.

Lemma 4. $|\Psi\rangle$ can be converted into $|\Phi\rangle$ by local unitary phase gates iff there exist phases $\left\{\bar{\alpha}_{i}\right\}_{i=0}^{n}$ such that $\left|\Psi_{\mathbf{0}}\right\rangle$ is converted into $\left|\Phi_{\bar{\alpha}_{i}}\right\rangle$ by local unitary phase gates.

Proof. If $|\Psi\rangle=e^{i \alpha_{0}} \bigotimes_{i=1}^{n} U_{i}\left(\alpha_{i}\right)|\Phi\rangle$ then choosing $\bar{\alpha}_{i}=\alpha_{i}$ for $i \in\{0, \ldots, n\}$ fulfills the condition. To prove the inverse direction we assume that there exist phases $\left\{\bar{\alpha}_{i}\right\}_{i=0}^{n}$ such that $\left|\Psi_{\mathbf{0}}\right\rangle=$ $e^{i \alpha_{0}} \bigotimes_{i=1}^{n} U_{i}\left(\alpha_{i}\right)\left|\Phi_{\bar{\alpha}_{i}}\right\rangle$ for some phases $\left\{\alpha_{i}\right\}$. Defining the projector $P=\sum_{\mathbf{k} \notin K}|\mathbf{k}\rangle\langle\mathbf{k}|$ we have $P\left|\Psi_{\mathbf{0}}\right\rangle=|\Psi\rangle$ and $P e^{i \alpha_{0}} \bigotimes_{i=1}^{n} U_{i}\left(\alpha_{i}\right)\left|\Phi_{\bar{\alpha}_{i}}\right\rangle=e^{i \alpha_{0}} \bigotimes_{i=1}^{n} U_{i}\left(\alpha_{i}\right) P\left|\Phi_{\bar{\alpha}_{i}}\right\rangle$ and therefore $|\Psi\rangle=e^{i \alpha_{0}} \bigotimes_{i=1}^{n} U_{i}\left(\alpha_{i}\right)|\Phi\rangle$.

Let us now use the lemma above to prove Lemma 2 .

Proof. Due to the lemma above it remains to show that for any state $|\psi\rangle$ with $\langle\mathbf{k} \mid \psi\rangle \neq 0 \quad \forall \mathbf{k}$ we have that $|\psi\rangle=e^{i \alpha_{0}} \bigotimes_{i=1}^{n} U_{i}\left(\alpha_{i}\right)|\phi\rangle$ iff condition (i) and (ii) in Lemma 2 are satisfied. Note that Eq. (2) is equivalent to $\langle 0 k \mid \psi\rangle\langle 1 l \mid \psi\rangle\langle 1 k \mid \phi\rangle\langle 0 l \mid \phi\rangle=$ $\langle 1 k \mid \psi\rangle\langle 0 l \mid \psi\rangle\langle 0 k \mid \phi\rangle\langle 1 l \mid \phi\rangle$, where 0,1 is acting on system $i$ and $k, l$ denote the computational basis states of the remaining $n-1$ qubits.

Let us now prove the only if part: If $|\psi\rangle=$ $e^{i \alpha_{0}} \bigotimes_{i=1}^{n} U_{i}|\phi\rangle$ then $\langle\mathbf{i} \mid \psi\rangle=e^{i \phi_{\mathbf{i}}}\langle\mathbf{i} \mid \phi\rangle$, with $\phi_{\mathbf{i}}=\alpha_{0}+$ $\sum_{k} \alpha_{k} i_{k}$, which implies (i). Condition (ii) (for $i=1$ ) is then equivalent to $e^{i\left(\phi_{0 k}+\phi_{1 l}\right)} x_{k l}=e^{i\left(\phi_{1 k}+\phi_{0 l}\right)} x_{k l}$, where $x_{k l}=\langle 0 k \mid \phi\rangle\langle 1 l \mid \phi\rangle\langle 1 k \mid \phi\rangle\langle 0 l \mid \phi\rangle$. It is easy to see that this condition is fulfilled since $e^{i\left(\phi_{0 k}-\phi_{1 k}\right)}=e^{-i \alpha_{1}} \forall k$. In 
the same way one can show that the conditions for $i \neq 1$ are fulfilled.

If: Condition (i) implies that $\langle\mathbf{i} \mid \Psi\rangle=e^{i \phi_{\mathbf{i}}}\langle\mathbf{i} \mid \Phi\rangle$, for some phases $\phi_{\mathbf{i}}$. Condition (ii) (for $i=1$ ) implies then that $e^{i\left(\phi_{0 k}-\phi_{1 k}\right)}=e^{i\left(\phi_{0 l}-\phi_{1 l}\right)} \forall k, l$, since $x_{k l}=\langle 0 k \mid \phi\rangle\langle 1 l \mid \phi\rangle\langle 1 k \mid \phi\rangle\langle 0 l \mid \phi\rangle \neq 0 \quad \forall k, l$. Thus, $e^{i\left(\phi_{0 k}-\phi_{1 k}\right)}$ must be independent of $k$ and therefore, we have $e^{i\left(\phi_{0 k}-\phi_{1 k}\right)}=e^{-i \alpha_{1}}$, or equivalently, $e^{i \phi_{k_{1}, k}}=$ $e^{i\left(\alpha_{1}^{\left(k_{1}\right)}+\phi_{1 k}\right)}$, where $\alpha_{1}^{(0)}=-\alpha_{1}$ and $\alpha_{1}^{(1)}=0$. Similarly

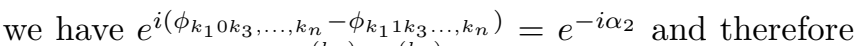
$e^{i \phi_{k_{1}, k_{2}, k_{3} \ldots, k_{n}}}=e^{i\left(\alpha_{1}^{\left(k_{1}\right)}+\alpha_{2}^{\left(k_{2}\right)}+\phi_{\left.11 k_{3}, \ldots, k_{n}\right)}\right.}$. Continuing in this way we find $e^{i \phi_{k_{1}}, \ldots k_{n}}=e^{i \alpha_{0}} e^{i \sum_{j} \alpha_{j} k_{j}}$, where $\alpha_{0}=$ $\phi_{1 \ldots 1}-\sum \alpha_{i}$. Thus, we have $|\psi\rangle=e^{i \alpha_{0}} \bigotimes_{i=1}^{n} U_{i}\left(\alpha_{i}\right)|\phi\rangle$ with $U_{i}\left(\alpha_{i}\right)=\operatorname{diag}\left(1, e^{i \alpha_{i}}\right)$. Using the lemma above, this implies that $|\Psi\rangle=e^{i \alpha_{0}} \bigotimes_{i=1}^{n} U_{i}\left(\alpha_{i}\right)|\Phi\rangle$.
[1] D. Gottesman, Ph.D. Thesis, quant-ph/9705052.

[2] R. Raussendorf and H. J. Briegel, Phys. Rev. Lett. 86, 5188 (2001).

[3] for a recent reviews see L. Amico et al., Rev. Mod. Phys. 80, 517 (2008); F. Verstraete et al., Adv. Phys. 57,143 (2008) and reference therein.

[4] see for instance V. Coffman et al., Phys. Rev. A 61, 052306 (2000); F. Verstraete et al., Phys. Rev. Lett. 92, 027901 (2004); J. Eisert and H. J. Briegel, Phys. Rev. A 64, 022306(2001).

[5] W. Dür et al., Phys. Rev. A 62, 062314 (2000); F. Verstraete et al., Phys. Rev. A 65, 052112 (2002).

[6] F. Verstraete et al., Phys. Rev. A 68, 012103 (2003).

[7] for a recent review see R. Horodecki et al., Rev. Mod. Phys. 81, 865 (2009) and references therein.

[8] M. Grassl et al., Phys. Rev. A 58, 1833 (1998).

[9] see for instance, S. Bravyi, Phys. Rev. A 67, 012313 (2003); J. Preskill, Lecture Notes for
Physics 219: Quantum Computation (California Institute of Technology, Pasadena CA 1998) URL: http://www.theory.caltech.edu/people/preskill/ph219/.

[10] C. Kruszynska and B. Kraus, Phys. Rev. A, 79, 052304 (2009).

[11] M. A. Nielsen, and I. L. Chuang, Quantum Computation and Quantum Information (Cambridge University Press, Cambridge, England, 2000).

[12] B. Kraus, in preparation.

[13] If there are less than $n$ linearly independent vectors in $S$, say $k$, then $k$ phases can be defined like that, the other phases leave the state invariant and can therefore be chosen arbitrarily.

[14] Note that this standard form coincides for the simplest case of two qubits with the Schmidt decomposition [11] and can be generalized to $d$-level systems.

[15] Note that the phases $\alpha_{i}$ can be easily computed. 